\title{
Étude de phénomènes physiques, de conception et de production de formes dans le champ de l'impression 3D céramique et du design
}

\author{
Studies of physical phenomena, design and production of forms in the
} field of ceramic 3D printing

\author{
Sophie Fétro ${ }^{1}$ \\ ${ }^{1}$ maître de conférences, Université Paris 1 \\ Panthéon-Sorbonne, UFR 04 Arts plastiques et sciences de l'art
}

RÉSUMÉ. Depuis 2009, date à laquelle le collectif de designers Unfold avec Tim Knapen développent L'Artisan Électronique, les projets et les machines d'impression 3D céramique se sont multipliées. Si dans le champ de l'industrie l'usage de poudre à base de céramique est plus ancien, jamais la mise en forme de la céramique n'avait été développée de cette façon, inaugurant des résultats inédits, laissant la part belle à l'aléa et à l'intégration de facteurs environnementaux. Soutenant bien souvent le partage libre des données, relevant elles-mêmes de l'autoproduction, elles interrogent la technique et les processus d'obtention des formes, développant une critique des approches industrielles propriétaires. Cette rencontre de l'impression 3D et de la céramique s'inscrit en particulier dans un revival naturaliste qui s'aventure aussi bien sur le terrain du biomimétisme, du biomorphisme, que du côté d'une écoute

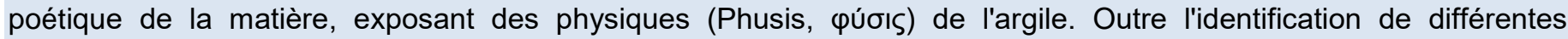
démarches de création et façons d'articuler l'impression 3D à la céramique, le propos porte également sur les raisons de leur convergence et sur un questionnement plus profond. L'hypothèse développée est que cette rencontre n'est pas sans lien avec les préoccupations actuelles relatives à l'écologie et l'anthropocène.

ABSTRACT. Since 2009, when the designer collective Unfold with Tim Knapen developed L'Artisan Électronique, ceramic 3D printing projects and machines have multiplied. If in the field of industry the use of ceramic-based powder is older, never the shaping of ceramics had been developed in this way, inaugurating unprecedented results, leaving room for randomness and the integration of environmental factors. Often supporting the open source, and themselves falling under the heading of self-production (DIY), they question the technique and processes of obtaining forms, developing a critique of proprietary industrial approaches. This meeting of 3D printing and ceramics is particularly part of a naturalist revival that ventures both into the terrain of biomimicry, biomorphism, and on the side of a poetic

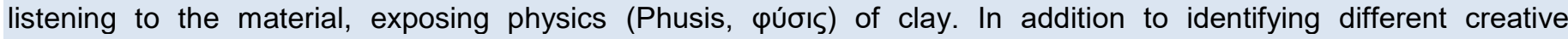
approaches and ways of articulating 3D printing to ceramics, the talk also focuses on the reasons for their convergence and on a deeper questioning. The hypothesis developed is that this meeting is not without a link to current concerns about ecology and the anthropocene.

MOTS-CLÉS. Impression 3D, céramique, open source, RepRap, design, terrestre, techno-esthétique, technophanie.

KEYWORDS. 3D printing, ceramics, open source, RepRap, design, terrestrial, techno-aesthetics, technophany.

\section{Exploration de phénomènes physiques et production de formes dans le champ de l'impression 3D céramique et du design}

Avec le développement des technologies de fabrication assistée par ordinateur, en particulier de l'impression 3D (fabrication additive), il est un domaine d'application spécifique qui a vu le jour ces dernières années : l'impression 3D céramique. Émergeant dans différents lieux, écoles, studios et ateliers de création, les machines qui l'ont rendu possible sont redevables en grande partie du développement d'internet et de l'open source, mais aussi d'un rapport artistique et critique aux outils et machines de production. Développées par des étudiants aussi bien que par des designers et artisans confirmés, elles ont en commun l'utilisation d'une matière commune : la céramique. Le développement industriel de l'impression 3D à base de poudre de céramique que l'on peut faire 
remonter aux années 80 (les premiers brevets datent de cette époque ${ }^{1}$ ), s'inscrit dans une logique de diversification et de substitution des matières plastiques employées aux débuts de la fabrication additive dans le secteur du prototypage rapide, motivé par la recherche de propriétés spécifiques et en quête de qualités nouvelles. Outre le fait qu'elle continue à s'inscrire dans le prolongement d'applications traditionnelles (sculpture, arts de la table), l'impression céramique trouve notamment dans l'industrie de pointe, le secteur du biomédical ${ }^{2}$ (dentisterie, prothèses, reconstruction osseuse), celui de l'industrie automobile, de l'aérospatiale, de la défense, des télécommunications (satellites, téléphonie), de l'énergie, de l'informatique et des composants électroniques, des terrains d'application spécifiques variées. La Recherche et Développement de ces secteurs de pointe conduit le plus souvent à améliorer la résistance et les performances de la céramique en agissant sur les structures moléculaires de la matière et en la combinant à d'autres matériaux (matériaux composites $^{3}$ ) en vue d'élargir ses propriétés physiques et ses possibles applications. L'impression céramique se caractérise par quatre technologies principales: le frittage de poudre ${ }^{4}$, la photopolymérisation $^{5}$, la pulvérisation de matière ${ }^{6}$ et l'extrusion ${ }^{7}$. Aujourd'hui les entreprises dédiées à l'impression 3D sont en nombre croissant et multiplient les dépôts de brevets afin de posséder des droits sur ces techniques. Les leaders de ce secteur se partagent les parts d'un marché en pleine expansion $^{8}$. Même s'il est indéniable que l'industrie a favorisé la productivité et des applications hautes performances de la céramique dans différents secteurs industriels ${ }^{9}$, ce n'est pas le développement de cette industrie sous l'angle d'une économie de marché et des technologies de haute précision, qui nous intéresse ici. Il est plutôt question de se pencher sur des productions et des démarches de création qui parviennent à faire exister un rapport artistique à ces techniques, sortant des habitudes productives courantes en intégrant dans les processus créatifs : conception paramétrique, programmation, code, création et transformation de machines. L'attention sera ici portée sur des production artistiques singulières, des artefacts qui opèrent une rencontre assez surprenante entre des technologies de pointe comme l'impression 3D et un art séculaire comme la

1 Le 1er juillet 1984 : dépôt du 1er brevet d'impression 3D par Jean-Claude André, Olivier Witte et Alain le Méhauté, pour I'entreprise CILAS ALCATEL. Le 1er août 1984, Chuck Hull dépose le brevet de la stéréolithographie (SLA), lequel lancera l'entreprise 3D Systems. À la fin de l'année 1988, la première imprimante 3D SLA-250 est lancée. Le frittage laser sélectif a été développé par Carl R. Deckard et Joseph J. Beaman dans les années 1980 à l'Université du Texas.

2 Des composites biocompatibles et/ou bioactifs sont utilisés dans le secteur médical.

3 Notamment des composites céramique-verre, céramique-polymère, céramique-métal.

4 Fusion couche après couche d'une poudre ici de céramique : SLS frittage sélectif de poudre au laser. "Malgré les limites connues du processus de densification pendant le SLS direct en raison du temps d'interaction court entre le laser et le matériau, la communauté céramique a montré un intérêt pour le processus SLS en raison de la possibilité de fabriquer des pièces aux formes complexes qui ne peuvent pas être produites à l'aide des processus traditionnels de mise en forme de la céramique. " Source : « Additive Manufacturing of Ceramic-Based Materials », par Nahum Travitzky, Alexander Bonet, Benjamin Dermeik, Tobias Fey, Ina Filbert-Demut, Lorenz Schlier, Tobias Schlordt and Peter Greil, dans Advanced Engineering Materials, 2014, 16, No. 6

5 Solidification d'un mélange de résine et de poudre céramique couche par couche sous l'action de la lumière.

6 Technique qui consiste à projeter des gouttelettes de liant sur une poudre céramique.

7 Procédé technique de compression de la matière passant à travers une filière. Cette technique sera celle développée majoritairement par les designers à partir d'imprimante 3D du type RepRap.

8 Source (Consultée le 10 décembre 2018) : https://www.3dnatives.com/impression-3d-ceramique-03102018/ 
poterie. Comment dès lors expliquer cette rencontre inattendue entre l'impression 3D et la céramique? Il faut sans doute chercher une hypothèse du côté des mutations que connait l'anthropocène et de ce que Bruno Latour appelle le «terreste ${ }^{10}$ » au regard des préoccupations actuelles : bouleversements climatiques, diminution de la biodiversité, dégradation des conditions de vie terrestres sous l'action avérée des activités humaines. Sans doute est-il nécessaire d'esquisser quelques hypothèses afin de comprendre cette inclination des designers a faire converger l'impression 3D et des processus de transformation de la matière semblables à ce que l'on peut appeler en biologie la morphogénèse. La terre qui renvoie davantage à la géologie et à l'essence physique même du monde semble bien éloignée des technologies de pointe de l'impression 3D qui recourt généralement à l'utilisation de matières plastiques (Polyéthylène), bien que des biopolymères comme le PLA à base d'amidon de maïs soient aujourd'hui largement employés. L'hypothèse soutenue est que l'impression 3D céramique s'inscrit dans ce que nous avons appelé un « Revival naturaliste ${ }^{11} \gg$. Les approches qui peuvent s'inscrire dans ce champ n'ont cependant ni seulement à voir avec ce que l'on appelle le biomorphisme (qui consiste à imiter les formes de la nature) ni exactement avec le biomimétisme (qui consiste à en imiter les processus), même s'il peut parfois en être question également de façon connexe, mais plutôt avec la mise en place de protocoles techniques spécifiques et des approches qui s'intéressent à l'observation attentive des phénomènes physiques ainsi qu'à l'étude du comportement de la matière. Il est alors moins question d'un rapport mimétique à la nature, que d'une attention portée à la physique même des choses et d'un questionnement sur la technique. L'impression 3D céramique semble ainsi déjouer les repères philosophiques et épistémologiques habituels, ne se situant ni dans l'opposition classique entre nature et culture ; ni tout à fait du côté de l'artisanat, ni tout à fait du côté de l'industrie ; mais dans un espace particulier, celui du design, qui précisément interroge ce qui peut exister dans l'espacement de ces polarités et tente d'opérer une rencontre possible et inattendue entre des technologies numériques et des matières argileuses issues d'un lent processus de sédimentation.

\section{Naissance de l'impression céramique}

Le premier exemple sur lequel il est possible de s'attarder et qui opère cette convergence de l'impression 3D et de la céramique est L'Artisan Électronique développé par le collectif belge Unfold en collaboration avec Tim Knapen, spécialisé en design d'interaction, pour l'exposition Design by Performance au centre d'art et de design contemporain Z33 à Hasselt, en Belgique. L'intitulé même du projet, L'Artisan Électronique, atteste de cette convergence des approches et moyens techniques, dépassant les clivages catégoriels habituels. Ce dispositif électronique de production de formes en céramique interroge non seulement l'artisanat dans ses pratiques et savoirfaire traditionnels, mais également le système productif industriel en série en faisant exister des modes d'autoproduction en dehors du circuit industriel conventionnel propriétaire fondé le plus souvent sur les dépôt de brevets et le secret. À travers cette suite ${ }^{12}$ de production faussement continue, les designers repensent ici l'ensemble du dispositif de production, impliquant l'usager dans la modélisation des pièces tout en explorant les potentialités esthétiques de ce complexe technologique. La finesse des résultats obtenus, le fait d'assumer l'esthétique du dépôt

10 Bruno Latour, Face à Gaïa. Huit conférences sur le nouveau régime climatique, Paris : La Découverte, 2015.

11 Sophie Fétro, "Revival naturaliste dans le champ de l'impression 3D », dans Formes vivantes, Milan : Silvana Editoriale S.p.A., 2019, p. 180-183.

12 Nous utilisons ici la notion de suite, renvoyant davantage à la suite musicale, et non celle de chaîne, afin de faire sentir la différence entre une chaîne de montage qui historiquement évoquer un enchainement optimisé et optimal d'actions continue (par exemple la ligne de montage automobile), et une succession de moments qui bien qu'ils aient des relations entre eux sont encore spécifiques, peuvent être arrêtés, lesquels acceptent des retours en arrière et à partir desquels l'intervention humaine est encore possible et préconisée. 
stratigraphique de la matière, la diversité des variations rendues possibles par l'informatique, attestent d'une approche sensible de la matière pour laquelle la technique n'est pas sollicitée à des fins d'efficacité productive et de rendement. Au fond, l'impression 3D céramique d'Unfold met en place une façon inédite de mettre en forme la matière et d'en révéler de nouveaux aspects jusqu'alors passés inaperçus. Les irrégularités, les petits accidents d'impression, la rugosité de surface, liés à l'extrusion de la matière, ne sont en rien, ou disons pas nécessairement, un problème dans la facture de l'objet ainsi produit, bien au contraire. Elles participent en réalité de l'identité matérielle (physique pourrions-nous dire) de l'artefact ainsi produit à travers lequel le mode opératoire transparaît. Claire Warnier, parlera à cet égard de «beauté de l'imperfection ${ }^{13}$ ». D'un point de vue de l'histoire de l'impression 3D céramique, 2009 constitue une date clé car ce sera la première fois que l'on verra une imprimante 3D de type RepRap capable d'imprimer à partir de composés à base d'argile. Unfold contribuera largement à la diffusion de cette nouvelle méthode de production céramique via la communauté RepRap «en aidant des artistes, des designers, des universités et des instituts du monde entier à configurer leurs propres imprimantes 3D céramique basées sur ce système ouvert ${ }^{14} »$, favorisant ce que Giorgio Agamben appelle à propos des dispositifs un processus de «profanation ${ }^{15} »$, au sens laïque du terme, c'est-à-dire une «restitution à l'usage commun ${ }^{16}$ ». Historiquement, L'artisan Électronique fait figure de précurseur dans le développement de l'impression 3D céramique au-delà d'ailleurs des champs artistiques et du design. À partir de cette date plusieurs designers vont se mettre à développer leurs propres machines et protocoles de mise en forme de la matière et, à travers eux, l'exploration de phénomènes physiques.

\section{Une approche artisanale de l'impression 3D céramique}

Tel est le cas de Jonathan Keep, autre figure marquante des débuts de l'impression 3D céramique. Proche d'Unfold ${ }^{17}$, ils participera d'ailleurs du développement de leurs outils et de la diffusion de leur processus d'impression : «En 2010, des imprimantes 3D en kit à monter soi-même sont arrivées sur le marché et un studio de design belge, appelé Unfold, a été le premier à fixer une seringue remplie d'argile à l'une de ces machines. Les imprimantes étaient jusqu'alors destinées à imprimer avec du plastique ${ }^{18}$. » Artisan de formation, ayant développé une carrière importante de céramiste, il s'est très tôt intéressé à l'informatique, explorant dès 2012 les interrelations entre le code et la terre, cultivant une familiarité avec les processus et les formes naturelles. Pour lui, grâce à l'informatique, à la programmation et au code « les traditions de la céramique viennent d'être actualisés ${ }^{19}$. » Sa série

13 Claire Warnier, L'Artisan Electronique, 2010. En téléchargement sur la page dédiée à L'Artisan Électronique (consultée le 29 juin 2021) : http://unfold.be/pages/l-artisan-electronique.html

14 " Over the past years Unfold has been actively diffusing this new method for producing ceramics by assisting artists, designers, universities and institutes worldwide to setup their own ceramic 3d printers based on our open system. " Source (consultée le 29 juin 2021) : http://unfold.be/pages/ceramic-3d-printing.html

15 Giorgio Agamben, Qu'est-ce qu'un dispositif ?, Paris : Payot \& Rivages, 2007. « La profanation est le contre-dispositif qui restitue à l'usage commun ce que le sacrifice avait été séparé et divisé. ", p. 40.

16 lbid., p. 50

17 II est indiqué dans le site web d'Unfold correspondant au descriptif du projet « L'artisan électronique » : « Avec Jonathan Keep, Unfold a initié Make Your Own Ceramic 3D Printer, une communauté en ligne dédiée au partage et au développement d'idées autour de l'impression 3d céramique. Avec la disparition de Google+ en 2019, la communauté a migré vers Wikifactory en ajoutant Tom Lauerman comme troisième administrateur. Un grand merci à l'équipe de WikiFactory pour avoir rendu cette transition possible. » Source (consultée le 29 juin 2021) : http://unfold.be/pages/ceramic-3d-printing.html

18 Jonathan keep, The form is in the code, source : http://www.keep-art.co.uk/texts Q\&A.html

19 Jonathan keep, The form is in the code, "Sur mon travail », 2013. Source : $\underline{\text { http://www.keep-art.co.uk/texts_Q\&A.html }}$ 
Iceberg, exposée au lors de la $3^{\text {ème }}$ Biennale de céramique britannique en 2013, témoigne de sont intérêt pour les phénomènes apparement aléatoires comme l'érosion de la glace et les procédés génératifs algorithmiques : «Le choix d'utiliser de la porcelaine blanche est également délibéré afin de faire écho à la translucidité de la glace - le processus, le matériau et le contenu de l'œuvre ne font qu'un. » Dans le champ de l'artisanat, Jonathan Keep fait toutefois figure d'exception. L'interêt qu'il a nourri pour l'impression 3D puise ses origines dans son travail informatique et la volonté de pouvoir faire sortir les modélisations numériques de leur caractère virtuel : «je me suis intéressé à l'impression 3D parce que je voulais sortir de l'ordinateur le travail que je réalisais informatiquement pour le faire entrer dans le monde physique sous forme d'objets tactiles et saisissables. L'impression 3D était précisément la technique qui le permettait (...) Mais au final, c'est la nature qui m'intéresse, ou notre relation à la nature. Travailler avec la technologie est le moyen par lequel je peux explorer cet intérêt pour la nature, la technologie est un moyen d'atteindre ce but ${ }^{20}$. $\gg$ Comme il le rappelle, la modélisation 3D et la fabrication additive ne sont pas concomitantes sur le plan historique, la visualisation 3D étant antérieure à l'impression 3D. La modélisation informatique et la CAO (Conception Assistée par ordinateur) ont en quelque sorte créé une attente de la part des créateurs : celle de voir se concrétiser leurs visualisations en sortant de la virtualité afin d'opérer le passage du fichier à la fabrication (ce que l'on appelle désormais couramment le «file to factory »). Bien que la fabrication soustractive puisse puiser ses origines dès le $19^{\text {ème }}$ siècle, avec des procédés tels que la photosculpture ${ }^{21}$, et que les machines-outils à commandes numériques ( $\mathrm{CN}$ et $\mathrm{CNC}$ ) existent de façon automatisée depuis les années 50 dans l'industrie ${ }^{22}$, l'impression 3D à proprement parler est plus récente et plus directement liée au développement du prototypage rapide ${ }^{23}$. «Le facteur déterminant pour l'impression 3D reposait

20 Jonathan keep, The form is in the code, source : http://www.keep-art.co.uk/texts Q\&A.html "In 2010 DIY kit 3D printers came onto the market and a Belgium Design Studio called Unfold where the first to attach a syringe filled with clay to one of these machines. The printers were intended to print with plastic. I visited Unfold and copied their technique and since then we have become good friends. So to answer your question, I became interested in 3D printing because I was generating this work in computer and wanted to get it out of the computer and into the physical world as objects you could pick up and feel, and $3 D$ printing offered the technique to do it. So for me it is about the way of working. I can do all the technology stuff but ultimately it is nature that interests me, or our relationship to nature. Working with technology is the means by which I can explore this interest in nature, the technology is a means to the end. The interest is not really in the technology although I accept it becomes part of the process and I am happy with that as I would like my ceramics to be an expression of my age, the digital age. " (2013) Traduction s. Fétro.

21 « Un photographe qui était aussi sculpteur, François Willème (1830-1905), partisan de l'art industriel, met au point une méthode combinant la photographie et la sculpture pour reproduire le réel. De 1863 à 1869, les Parisiens ont pu s'offrir, grâce à la Société générale de photosculpture, leur buste sculpté en un temps record pour un prix record ; Le modèle se tenait exactement au centre d'une rotonde de dix mètres de diamètre, le temps que vingt-quatre appareils photographiques disposés à égale distance réalisent simultanément vingt-quatre clichés. Puis les profils obtenus étaient projetés par un lampascope sur un écran transparent, à la taille de la sculpture souhaitée. Le profil projeté était ensuite reporté au moyen d'un pantographe sur un bloc de glaise. L'addition de l'ensemble des profils restituait avec une grande exactitude l'apparence du modèle. " Dans Sylvain BELLENGER, «PHOTOSCULPTURE », Encyclopædia Universalis [en ligne], consulté le 29 juin 2021. URL : http://www.universalis-edu.com/encyclopedie/photosculpture/

22 Richard KEGG, en collaboration avec le MIT, développe la première fraiseuse à commande numérique par calculateur : la Cincinnati Milacron Hydrotel, en 1952, et dépose en 1958 le brevet pour les « Motor Controlled Apparatus for Positioning Machine Tool», ouvrant la voie à l'application commerciale de cette technologie. Source (consultée le 29 juin 2021) : https://www.manufacturier.quebec/usinage-CNC

23 Claire Warnier indique cependant à propos de l'impression 3D et de sa relation au prototypage rapide que la rapidité reste cependant toute relative : "This technique is called 3D printing, also known as Rapid Prototyping or Rapid Manufacturing. Although in fact, there is nothing rapidabout it. In order to print a $10 \mathrm{~cm}$ object, the machine takes about an hour. It's soothing to watch, however. With each new layer, the humming motors sing to a more or less identical tune. " (traduction : "Cette technique est appelée impression 3D, également connue sous le nom de prototypage rapide ou de fabrication rapide. Mais en 
donc sur mon travail numérique. Je créais mon travail sur ordinateur et le seul moyen de le sortir de l'ordinateur consistait à utiliser la technique de l'impression $3 \mathrm{D}^{24}$. " Pour Jonathan Keep, l'impression 3D céramique constitue une $4^{\text {ème }}$ méthode ${ }^{25}$ de mise en forme de la céramique venant s'ajouter aux trois techniques déjà existantes: le modelage manuel (enroulement de colombins, construction par plaques, modelage et façonnage à la main), le moulage et le tournage. Elle n'est en rien incompatible avec ces autres techniques, offrant plutôt de nouvelles possibilités créatives et une diversification des moyens de mise en forme de la matière. En dehors du champ de la céramique, la rencontre des technologies numériques avec des phénomènes et formes naturelles s'opèrent également. Des expositions comme Naturaliser l'architecture ${ }^{26}$, En Vie/Alive ${ }^{27}$, La fabrique du vivant $^{28}$, au dernièrement Formes vivantes ${ }^{29}$ au musée Adrien Dubouché de Limoges, pour ne citer que quelques expositions en France, attestent depuis une dizaine d'années de l'intérêt des artistes, des designers et des architectes, pour le vivant, la «nature » et les phénomènes physiques en général dans leur rapport aux technologies. Ce regain d'intérêt ne doit en réalité rien au hasard. Il est indéniablement corrélé à une préoccupation, voire une inquiétude, quant aux mutations actuelles, mais aussi à une volonté de comprendre la terre, en tant que phénomène actif, ainsi que les interactions entre les êtres et les choses qui la constituent.

\section{Explorer le potentiel expressif de l'impression 3D avec l'industrie}

D'autres designers explorent le potentiel expressif et artistique de l'impression 3D céramique. Francesco Pacelli, artiste et designer diplômé de l'École Polytechnique de Milan en conception et ingénierie (2013), et chercheur au LAB+ (laboratoire d'impression 3D de la Politecnico de Milan), s'est associé à la société WASP, entreprise italienne spécialisée dans l'impression 3D grand format.

fait, elle n'a rien de rapide. Pour imprimer un objet de $10 \mathrm{~cm}$, la machine met environ une heure. Mais c'est apaisant à regarder. À chaque nouvelle couche, le bourdonnement des moteurs chantent une mélodie plus ou moins atonale. ") dans Claire Warnier, L'Artisan Électronique, op. cit.

24 "So the driving factor to go down the route of 3D printing was because I was working digitally. I was creating my work on computer and the only way to get it out of the computer was with the technique of 3D printing. ", Jonathan Keep. Proposition de traduction : « Le facteur déterminant pour l'impression 3D était donc mon travail numérique. Je créais mon travail sur ordinateur et le seul moyen de le sortir de l'ordinateur consistait à utiliser la technique de l'impression 3D. " Source (consultée le 29 juin 2021) : http://www.keep-art.co.uk/texts Q\&A.html

25 A propos de l'impression 3D Céramique Jonathan Keep indiquera : "It will not become the dominant forming technique but it will be added to the techniques we have, offering a fourth way - moulding, throwing, hand building and then printing. (Current technical problems are scale, supporting of forms while printing and surface quality). " (2013). Traduction : " Elle ne deviendra pas la technique de mise en forme dominante, mais viendra s'ajouter aux techniques existantes, offrant une quatrième méthode : moulage, tournage, modelage manuel, puis impression 3D. (Les problèmes techniques actuels restent : l'échelle, l'effondrement de la matière lors de l'impression et la qualité des surfaces). "

26 Archilab 2013 : Naturaliser l'architecture (9ème édition), Frac Centre d'Orléans, exposition du 14 septembre 2013 au 02 février 2014. Commissaires : Marie-Ange Brayer, Frédéric Migayrou. Assistance au commissariat. Emmanuelle Chiappone-Piriou. Frac-Centre, Orléans. Catalogue d'exposition : Naturaliser l'architecture / Naturalizing architecture, Orléans : éditions HYX, 2013.

27 En Vie, aux frontières du design / Alive, new design frontiers, Fondation EDF, exposition du 26 avril au 1 er septembre 2013. Commissaire : Carole Collet. Catalogue d'exposition : En Vie, aux frontières du design / Alive, new design frontiers (dir. Carole Collet), Fondation EDF, Londres : Push, 2013.

28 La fabrique du vivant, dans le cadre de Mutations/Créations 3, Centre Pompidou, Galerie 4, Paris, du 20 février au 15 avril 2019. Catalogue : La Fabrique du vivant (dir. Marie-Ange Brayer, Olivier Zeitoun), Orléans : HYX, 2019. 
Au sein de cette entreprise, son travail a consisté à montrer le potentiel et les limites du LDM (Liquid Deposition modeling soit le dépôt de matière liquide), à déterminer le mélange approprié de la matière employée, à définir les paramètres d'impression associés et à élargir les types d'objets susceptibles d'être ainsi imprimés avec cette technologie. Les céramiques qu'il a produit dans ce cadre sont le résultat du partenariat avec cette entreprise mais aussi avec un atelier de céramique traditionnel situé à Faenza en Italie (ville réputée depuis la Renaissance pour ses céramiques et connue pour avoir donné l'appellation faïence en français). Mangrove métallique qui ressemble à un système racinaire, que l'on pourrait difficilement obtenir autrement que par impression 3D au regard de la spécificité de la pièce et de ses formes intérieures, revêt un aspect biomorphique. Le designer a eu recours à une technique traditionnelle de vitrification développée à l'époque de la Renaissance appelée la "réduction plein feu" qui permet d'obtenir un émaillage très fin avec des reflets irisés, ce que l'on appelle aussi la majolique. Francesco Pacelli indique à cet égard vouloir « établir un lien historique entre les méthodes de fabrication contemporaines de l'impression 3D et les métiers traditionnels de la céramique ${ }^{30} »$. Mangrove métallique ou Piromania, une autre de ses productions qui revêt un côté plus inquiétant, peut-être du fait de sa couleur rouge, célèbrent à leur manière la rencontre de puissances et forces a priori divergentes : les éléments (la terre, l'eau, l'air, le feu), l'informatique, le calcul, la mécanique relative à la machine d'impression 3D, associés à des réactions chimiques et des phénomènes physiques. Ces artefacts qui ont subi de multiples transformations, traduisent bien l'esprit d'une époque tiraillée par des aspirations contradictoires : celle, d'une part, qui pousse du côté global et d'une approche progressiste du développement technologique, si l'on suit l'approche de Bruno Latour ${ }^{31}$, et, d'autre part, qui conduit du côté du local et d'un ancrage à la fois culturel et historique.

\section{Un rapport de contingence à l'impression 3D et à la matière}

Avec Mesdames Grès, Laure Giraudaud, s'intéresse davantage à la matérialité même de la terre : à sa physique, pourrait-on dire. Exposée au sein de l'exposition L'arbre de Darwin. La céramique comme expérience, ${ }^{32}$ les différentes pièces qui composent cette approche expérimentale de l'impression 3D, montrent des affaissements volontairement incontrôlés de la matière. Il en ressort des vases aux formes irrégulières, issues de l'effondrement de la matière lors de l'impression 3D. Au lieu de créer des supports pour contrer ce phénomène bien connu dans le champ de l'impression 3D, la designer a préféré laisser faire la matière, générant des débordements et proéminences qui ne sont pas sans évoquer ce qui pourrait être considéré, transposé dans le champ de la botanique, comme des anomalies végétales ${ }^{33}$. Pourtant, au-delà de la comparaison, la nature n'est ici ni prise comme modèle formel, ni prise comme modèle structurel ou génératif. Il est plutôt question ici de la plasticité de la terre, du phénomène en tant que tel, qui est exposé montrant le caractère actif de la terre. Ce principe physique d'effondrement de la matière est ici pleinement assumé et participe de l'identité même des pièces. Laure Giraudaud tire parti de ces évènements impromptus et produit des pièces qui tiennent compte du poids de la matière, de l'attraction terrestre, pour en tester le potentiel

30 Francesco Pacelli, Bilinski, 2015. Source (consultée le 29 juin 2021) : http://futureartistmakers.org/artists/francesco-pacelli

31 Bruno Latour, Où atterir ? Comment s'orienter en politique, Paris : La Découverte, 2017.

32 L'arbre de Darwin. La céramique comme expérience. Exposition du 28 juin au 3 novembre 2018, prolongation jusqu'au 1er décembre 2018. Cette exposition est réalisée dans le cadre d'un partenariat avec l'ENSA Limoges. Commissariat : Michel Paysant, plasticien, en charge de la structuration de la recherche et responsable du laboratoire de recherche CCE (La céramique comme expérience), l'équipe CCE, Guy Meynard et Arnaud Borde, à l'ENSA Limoges.

33 Théophraste, botaniste grec ( $3^{\text {ème }}$ siècle Av. J.-C.), dans Les causes des phénomènes végétaux (Livres V et VI), s'intéressait déjà aux morphologies des plantes, aux anomalies et accidents présents dans la nature, " aux phénomènes dont les arbres et les plantes en général sont le siège, les uns se produisent spontanément, les autres relèvent d'un aménagement cultural ». 
formel et esthétique. Au lieu de les considérer comme une entrave à la réalisation d'une pièce, ces déformations, ces collapses, sont générateurs de formes. Cette approche hylémorphique de l'impression 3D pourrait être qualifiée de contingente, en référence à la philosophie aristotélicienne. L'hylémorphisme dont il est question ici ne consiste pas à surimposer une forme à la matière, à seulement l'informer numériquement, mais à interroger les interrelations entre la technique (l'impression 3D), la matière (la céramique), les caractéristiques du milieu et les formes qui en découlent, sans hiérarchie les unes par rapport aux autres, plutôt dans un rapport dynamique et de réciprocité entre elles tout en acceptant que des accidents, des évènements inopinés, surviennent. Cette ouverture, cette écoute de la matière et de la technique, donnent la part belle à l'imprévu et implique, dans le cas qui nous intéresse ici, de ne pas tout préméditer à l'avance. Le travail du designer ne consiste pas alors à définir préalablement une forme, à la programmer et à l'imposer à la matière mais plutôt à mettre en place les conditions d'émergence et de survenue de phénomènes physiques inattendus à même de générer de la surprise ce qui pour Aristote constitue l'une des conditions même du caractère poétique d'une oeuvre. S'il y a bien un travail de programmation numérique de la machine, et donc la présence d'un protocole fortement réglé, son pilotage ne définit pas tout ce dont il est possible qu'il advienne : une part est laissée au hasard. Cet intérêt pour la surprise et les accidents heureux se retrouve chez d'autres designers. Olivier van Herpt revendique clairement son intérêt pour l'aléa et des facteurs qu'il ne souhaite pas entièrement contrôler. Designer hollandais, qui a mis au point ses propres machines d'impression 3D grand format depuis 20122013, il a développé une approche expérimentale de la matière : "En introduisant des éléments aléatoires, dit-il, j'ai voulu réintroduire de l'erreur, une part d'humain, de la stochastique. Je sentais que le processus avait besoin d'un peu de sérendipité, de joie à travers une sorte d'échec intentionnel. Je voulais de la répétabilité et de la précision, mais je me suis aperçu que j'avais aussi besoin d'erreurs $^{34}$. » Son approche montre que le travail du designer consiste avant toute chose à mettre en place les conditions d'apparition de phénomènes inattendus et a constituer à partir d'eux une sorte de grammaire. Citons également le travail de Rael San Fratello qui a développé avec l'université de Berkeley toute une série de productions intitulée GCODE.clay. Très proche du travail mené par Olivier van Herpt, il explore le potentiel créatif de la conception paramétrique à partir de différentes matières céramiques. Il utilise pour cela un logiciel qu'il a mis au point et qui lui permet de faire varier les paramètres de chaque modélisation. Il indique que ce qui l'intéresse ce sont les «séries d'erreurs contrôlées qui créent une nouvelle expression dans l'argile définie par la plasticité du matériau, la gravité et le comportement de la machine ${ }^{35}$.» Notons que si plusieurs designers plébiscitent cet intérêt pour l'aléa et l'imprévu, ce n'est toutefois pas le cas dans tous les secteurs.

\section{Une approche « contre nature ${ }^{36} »$}

Dans le champ de l'industrie de l'impression 3D à base de résines et de matières plastiques notamment, ces phénomènes de déformation de la matière sont généralement considérées comme

34 Olivier van Herpt : «By introducing elements of randomness I wanted to reintroduce error, a human touch, stochasticity. I felt that the process craved some serendipity, joy through intentional failure. I wanted repeatability and precision but found I also needed mistakes. » Source (consultée le 29 juin 2021) : https://oliviervanherpt.com/3d-printing-ceramics/

35 Rael san Fratello, GCODE.Clay, 2016 : «In this case the 3D printer is pushed outside the boundaries of what would typically define the printed object, creating a series of controlled errors that create a new expressions in clay defined by the plasticity of the material, gravity and machine behavior. » Source (consultée le 29 juin 2021) : https://www.rael-sanfratello.com/

36 Cette idée renvoie directement aux « phénomènes contre nature » observés par le philosophe et botaniste grec Théophraste. Cette formule désigne des phénomènes qui « relèvent, dit-il, d'une technique culturale, l'un est destiné à seconder la nature pour la qualité et le nombre des fruits, l'autre vise à obtenir des fruits particuliers et extraordinaires, par exemple un raisin sans pépins, ou du raisin noir et du raisin blanc sur le même serment. " Dans Théophraste, Les causes des phénomènes végétaux, Livres V et VI, Paris : Les Belles Lettres, 2017, Livre V, 1.1. 
des erreurs ou des malfaçons qu'il convient d'atténuer ou d'éliminer. Il s'agit alors de contrer ces phénomènes, de réduire les épaisseurs de matière pour éviter de voir les strates de dépôt de matière afin d'obtenir des pièces les plus conformes au modèle numériques et généralement les plus lisses possibles avec un degré de résolution le plus élevé possible ${ }^{37}$. Tel est le cas dans le champ de l'industrie médicale ou de l'aérospatiale, secteurs qui cherchent à produire des pièces à haut degré de précision. Des entreprises comme Formlabs ${ }^{38}$ qui se sont spécialisées dans la production d'imprimantes 3D et d'outils d'aide à la conception proposent des logiciels permettant de contrer les phénomènes de déformation et d'effondrement de la matière, d'identifier les zones de fragilité des pièces afin de définir les zones qui nécessitent des supports. Ces supports sont ensuite enlevés après impression conduisant à un travail de post-production parfois important consistant à les enlever et à lisser les points de contact. Contrairement aux machines des makers et des designers vues précédemment, les machines industrielles ressemblent plutôt à des volumes monoblocs desquels sortent, derrière un capot, des pièces obtenues sans que leur fonctionnement soit visible, accessible et intelligible. Cette approche «contre nature » de l'impression 3D, qui est bien souvent celle développée par l'ingénierie et l'industrie, est bien différente de l'approche contingente évoquée précédemment, car elle cultive un certain mystère à l'égard des phénomènes physiques et des procédés techniques. Gilbert Simondon les qualifierait de « $\operatorname{cryptotechniques}^{39} »$, car les objets qui en découlent ne disent en rien comment ils sont obtenus et dissimulent leur genèse.

\section{Une approche «épiphanique » de l'impression 3D : exposer les artifices employés}

À l'inverse de cette approche «cryptotechnique», certains artistes vont au contraire choisir d'exposer délibérément les processus techniques auxquels ils ont recours. Tel est le cas d'Eva L'hoest, qui propose de rendre manifeste ces artifices et éléments de support. Bien qu'il soit encore question d'un approche hautement artificielle qui consiste à pallier à l'effondrement de la matière (donc à contrer artificiellement la «nature », disons le phénomène physique d'affaissement de la matière ductile), l'approche consiste à exposer les artifices employés : au lieu de retirer ces dispositifs d'échafaudage, l'artiste les intègre comme partie prenante de son installation et en révèle l'existence tout autant que la beauté. Son oeuvre Shitsukan of Objects, qui signifie littéralement « le procédé des objets », rejoint un autre concept simondonnien, celui d' «épiphanie esthétique ${ }^{40}$ ». Contrairement à l'approche cryptotechnique qui conduit à dissimuler la technique, l'approche épiphanique à l'inverse l'expose. Cette œuvre qui pourrait également être qualifiée de « phanérotechnique ${ }^{41} »$ ne dissimule pas la façon dont les formes ont été obtenues : elle la manifeste, de sorte que le sujet représenté n'est plus seulement ici un chien mais l'échafaudage qui a permis de réaliser techniquement cette figure, autrement dit l'artifice lui-même. Bien qu'il ne soit pas ici question d'impression 3D céramique, la question de l'exposition des modes d'obtention des formes et de façon plus générale de la technique, se pose.

37 Plus la résolution est élevée, plus les couches de matière imprimées seront fines, moins les strates seront visibles.

38 Source consultée le 29 juin 2021 : https://formlabs.com/fr/

39 Gilbert Simondon, Sur la technique (1960-61), Paris : Presses Universitaires de France, 2014, p. 37-39.

40 Gilbert Simondon, Du mode d'existence des objets techniques, Paris : Aubier, 1958, 1969, 1989, p. 185. « Tout objet technique, mobile ou fixe, peut avoir son épiphanie esthétique, dans la mesure où il prolonge le monde et s'insère en lui. ॥ 
Pour revenir à l'impression 3D céramique, le dispositif technique mis au point par les designers Sander Wassink et Olivier van Herpt intitulé «adaptive manufacturing ${ }^{42}$ » a été pensé pour être réceptif à son contexte. Bien qu'ils ne détaillent pas exactement la nature de leurs scripts, ils indiquent que certaines informations externes sont mesurées par des capteurs, qui sont ensuite traduites en comportements via un logiciel, puis transmises à l'imprimante 3D qui les restitue à travers l'objet imprimé, un peu à la façon des cernes d'un arbre révélant la croissance végétale ou d'un sismographe témoignant des caractéristiques environnementales du milieu dans lequel la machine se trouve. Son titre «adaptive manufacturing», littéralement en français «fabrication adaptative », a pour but d'introduire des données locales dans le dispositif de production. Faisant le constat que «nous avons perdu la traduction des influences locales dans nos produits ${ }^{43}$ », les deux designers proposent une machine capable de détecter l'environnement proche et d'intégrer ces facteurs contextuels dans le processus de production, tout en maintenant le pouvoir décisionnel de l'être humain qui continue de pouvoir infléchir le résultat, de sélectionner certaines informations plutôt que d'autres.

\section{Un rapport encore poétique à la Terre}

Pour conclure, je dirais que ces designers, en s'intéressant à la fois à la céramique et aux technologies numériques opèrent non pas un « retour à la terre », mais ce que Bruno Latour appelle « un retour de la Terre ${ }^{44} »$, en faisant converger la modernité des technologies et le terrestre. Ces productions sont sans aucun doute une façon d'interroger l'anthropocène, l'occasion de permettre la manifestation d'un hiatus entre culture et nature, entre les technologies actuelles de dernière génération et les préoccupations pour le vivant et l'écosystème. Pour autant, les technologies et les moyens mis en œuvre par ces designers-makers, ne sont pas ceux directement issus de la grande industrie. Ces machines «profanes » qui ont été fabriquée librement en autoproduction, impliquent, à côté de l'industrie propriétaire, un bricolage des machines, des adaptations, le recours à des moyens lowtech, en lien avec des réseaux de makers, s'inscrivant dans le contexte de l'open-source et du partage libre des données, impliquant l'usage de moyens techniques détournées de leurs usages premiers et adaptées à la céramique. Ces démarches sont particulièrement intéressantes car, d'une part, elles ne rejettent pas les technologies, d'autre part, elles ne les sollicitent pas dans la démesure. Elles posent ainsi comme possible un rapport créatif, possiblement dissonant, à la technique, pour lequel l'accident n'est pas la catastrophe, la technique n'est ni conquérante ni arrogante, la terre est considérée dans sa contingence même, sa fragilité, envisagée comme un trésor plutôt que comme fonds ${ }^{45}$. Autrement dit, ces approches n'encouragent ni la perpétuation de modèles déjà admis

\footnotetext{
42 Sander Wassink et Olivier van Herpt, Adaptive Manufacturing, 2014.
}

43 Sander Wassink et Olivier van Herpt, Adaptive Manufacturing. A Sensory Machine That Feels Its Environment, $2014-\mathrm{en}$ cours. Descriptif du projet : "When we replaced the craftsmen by machines we lost the translation of local influences into our products. » Source consultée le 29 juin 2021 : https://oliviervanherpt.com/adaptive-manufacturing/

44 Bruno Latour, Où atterrir ? Comment s'orienter en politique, Paris : La découverte, p. 72. « II ne faut pas confondre le retour de la Terre avec le « retour à la terre » de triste mémoire. »

45 Martin Heidegger, Essais et conférences, Chap. I « La question de la technique ", Paris : Gallimard, 1958, p. 9-48. II serait d'ailleurs nécessaire d'interroger le rapport à la terre autrement que sous l'angle du dévoilement heideggerien et de l'arraisonnement de la nature. Quelle alternative imaginer au concept de fonds élaboré par Heidegger ? II s'agit ainsi de dépasser l'idée de nature comme pourvoyeuse de ressources dont il est alors question. De même que la notion de gisement pourtant admise dans les approches écologiques, serait également à interroger et à contester. Cette dernière qui renvoie à l'univers de l'exploitation minière, répète en réalité la notion heideggerienne de fonds. À celle-ci, lui seraient sans doute préférables les notions de grenier ou de trésor, qui impliquent un soin, une économie, une attention portée à sa forme et son contenu. 
(approche conservatrice) ou un retour au passé (approche rétrospective), ni une fuite en avant (occultant la réalité et les urgences à traiter), elles proposent plutôt de réfléchir à l'état du monde actuel, à des rapports singuliers à la technique en quête d'un rapport mesuré au monde capables d' interroger leur entame ${ }^{46}$ sur le terrestre. Ici, les designers et artistes ne se situent pas dans un « orgueil de domination» technocratique (Simondon) face à une matière qu'il faudrait dompter, contenir et maîtriser, mais dans une écoute attentive et patiente. Ils font cette tentative d'opérer la rencontre du numérique et de la matière physique, du code et de la matière, du calcul et de la contingence, de la programmation et de l'imprévu. C'est peut-être en cela que ces approches pourraient constituer, sans doute plus qu'il n'y parait, un modèle tangible et possible, c'est un tout cas l'hypothèse que je fais, pour un rapport non totalement déréglé ou perfide à la nature, mais mesuré et encore poétique à la technique et à la terre.

\section{Eléments biographiques}

Sophie Fétro est enseignante-chercheuse, théoricienne du design, maître de conférences à l'Université de Paris 1, spécialiste des questions de design et de ses théories. Elle développe une recherche qui porte sur les outils de conception et les modalités opératoires des designers, notamment lorsqu'ils impliquent des machines numériques et des programmes informatiques. Elle est actuellement membre du programme de Recherche ANG-G (ANR) et responsable artistique et scientifique pour la partie design de l'archive numérique Collecta (https://www.collecta.fr/). Elle produit des essais pour divers catalogues d'exposition (Imprimer le Monde, Centre Pompidou, 2017; Design et merveilleux, MAMC Saint-Etienne, 2018 ; Formes vivantes, Musée National Adrien Dubouché de Limoges, 2019), a été commissaire de l'exposition Design ex-machina ! à la galerie Michel Journiac (EAS, Paris, 2015) et a organisé le séminaire «Actualité du Bauhaus. Célébration d'un centenaire! » (École des Arts de la Sorbonne, 2019). 